\title{
Inmigración ilegal y actividad económica agregada en Estados Unidos: un análisis de series de tiempo
}

Carlos Heriberto flores Orona*, Ramón a. Castillo PONCE**y MARÍa de LOURdes ROdRÍguez EsPINOSA***

\section{RESUMEN}

En este documento se evalúa la relación que existe entre los flujos de inmigración ilegal y la actividad económica agregada en Estados Unidos. El análisis considera los efectos de corto y largo plazos. Las variables económicas incluyen el PIB nacional, el PIB de los estados que albergan el mayor número de inmigrantes indocumentados, y el empleo en las industrias donde labora una cantidad significativa de inmigrantes. Los resultados indican que, tanto en el corto como en el largo plazos, la variable de inmigración ilegal no muestra un efecto negativo y significativo sobre el desempeño económico de los estados e industrias analizadas.

Palabras claves: inmigración ilegal; actividad económica; empleo; Estados Unidos.

Clasificación JEL: F22, C32.

\footnotetext{
* Profesor-investigador, Instituto Tecnológico y de Estudios Superiores de Monterrey, México. Correo-e: chflores@tec.mx

** Profesor-investigador, California State University, Los Angeles, EE. UU. y Universidad Autónoma de Baja California, México. Correo-e: rcastil@calstatela.edu2

${ }^{* * *}$ Profesor-investigador, Universidad Tecnológica de la Mixteca, México.

Correo-e: mdlrodrig@mixteco.utm.mx
} 


\section{ABStRACT \\ Illegal Immigration and Aggregate Economic Activity in the United States: A Time Series Analysis}

This document evaluates the relationship between the flows of illegal immigration and the economic activity in the United States at the aggregate level. The analysis considers the long and the short terms. Economic variables include national GDP, GDP for the states where a large number of illegal immigrants reside, and employment for industries with significant participation of undocumented workers. The results suggest that neither in the short nor in the long term, illegal immigration presents a negative and significant effect on the economic performance of the states and industries analyzed.

Keywords: Illegal Immigration, Economic Activity, Employment, United States of America

JEL classification: F22, C32.

\section{INTRODUCCIÓN}

A pesar de que el tema de inmigración en Estados Unidos ha tomado particular importancia en tiempos recientes, el debate no es nuevo. Por décadas, círculos académicos, políticos y sociales en ese país han discutido el efecto de este fenómeno sobre la dinámica socioeconómica de sus comunidades. Por un lado, de manera anecdótica, se argumenta que el flujo de inmigrantes, legales o ilegales, produce condiciones sociales y económicas desfavorables. Frecuentemente se hace referencia al incremento en el crimen, al abuso de programas de asistencia social y otros males sociales que se le atribuyen a la inmigración. En tiempos recientes, esta percepción negativa incrementó sustancialmente. La administración federal en Estados Unidos, gestión correspondiente a 2017, se encargó de estigmatizar a los inmigrantes y el resultado de su campaña antiinmigrante se percibe en la cobertura sesgada sobre el tema de medios de comunicación conservadores. Basta sintonizar las noticias de la cadena FOX para constatar este hecho. Sin embargo, se reconoce que los inmigrantes acarrean beneficios a las comunidades que los reciben. Típicamente son personas que siguen las leyes más estrictamente que quienes son nativos del país; los inmigrantes son 
personas trabajadoras, dedicadas y perseverantes, que se sobreponen a condiciones adversas y salen adelante. Hay industrias, como la restaurantera y de servicios de hospitalidad, que dependen fundamentalmente de la mano de obra inmigrante. Sin duda, esta divergencia en opinión y percepción de la inmigración no será reconciliada en tiempos próximos, al menos no mientras el gobierno federal de Estados Unidos no cambie radicalmente su postura hacia los inmigrantes.

A decir verdad, la falta de acuerdo en cuanto al efecto neto de la inmigración no es sólo un asunto de opinión o percepción; investigaciones en diversas ciencias sociales tampoco han podido alcanzar una postura unificada. En la ciencia económica, la falta de acuerdo es igualmente evidente. Investigaciones sobre cómo los flujos de migración "ilegal" afectan la actividad económica abundan y sus resultados no apuntan hacia un consenso. Por señalar algunas, podríamos mencionar aquellas que se centran en analizar el mercado laboral. Al respecto, Borjas $(2003 ; 2011)$ destaca la caída de las oportunidades de trabajo y salarios para los trabajadores nativos no especializados, así como la elevada incidencia en el uso de programas de ayuda o welfare por parte de los residentes que no cuentan con documentación migratoria válida.

En contraste, Altonji y Card (1991) y Ottaviano y Peri (2012) encuentran que la inmigración no produce resultados significativamente negativos sobre los salarios. Esto es explicado en Peri y Sparber (2009), quienes identifican un cambio en la especialización de trabajadores estadounidenses de tareas manuales a tareas enfocadas en habilidades de comunicación, mientras que los inmigrantes continúan empleándose en trabajos intensivos en mano de obra. Peri (2016) provee una amplia discusión contemporánea sobre el tema. Claramente el debate sobre los costos y beneficios de la inmigración en el ámbito académico continuará.

De esta manera, el propósito del presente documento no es identificar el efecto neto que la inmigración presenta sobre las comunidades receptoras, sino evaluar de manera formal la relación estadística entre los datos de inmigración, particularmente ilegal, y la actividad económica agregada en Estados Unidos. En este sentido, hacemos una pausa en la corriente de análisis e investigamos el fenómeno en un nivel más primitivo, es decir, de origen. ¿Qué relación estadística existe entre el desempeño económico y la inmigración ilegal? Ésta es la pregunta que nos interesa responder. El acercamiento metodológico que empleamos 
se enmarca en el contexto de series de tiempo; en este aspecto difiere de los estudios que típicamente se encuentran en la literatura y que consideran datos desagregados, incluyendo observaciones de sección cruzada o datos de panel. Notamos, sin embargo, que el análisis con series de tiempo es consistente con lo que estudios sobre inmigración ilegal han empleado anteriormente. Hanson y Spilinbergo (1999), por ejemplo, analizan los factores que determinan la inmigración ilegal hacia Estados Unidos. Los autores postulan las aprehensiones de migrantes en la frontera México-Estados Unidos como variable dependiente, y una serie de variables explicativas que incluyen el diferencial salarial entre los dos países y las horas que la patrulla fronteriza vigila la frontera. Los resultados sugieren que las diferencias salariales representan un factor fundamental en la dinámica migratoria. Similarmente, Cornwell (2009) investiga cómo la vigilancia en la frontera influye sobre los flujos de inmigración ilegal. El autor concluye que el número de aprehensiones se encuentra correlacionado con las horas de vigilancia. Sin embargo, las aprehensiones no ayudan a pronosticar la vigilancia que habrá en el futuro, o viceversa. Por su parte, Mandelman y Zlate (2012) estiman un modelo de equilibrio general estocástico dinámico (DSGE, por sus siglas en inglés) en el que analizan la relación entre migración y remesas dentro del marco de los ciclos económicos de México y Estados Unidos. El modelo emplea series de tiempo en el espíritu de los dos documentos mencionados, que incluyen el número de aprehensiones y las horas de vigilancia fronteriza. Los autores encuentran que las barreras a la migración producen un efecto asimétrico sobre el bienestar de los trabajadores calificados y no calificados.

Más allá de emplear un acercamiento metodológico escasamente utilizado en el estudio del fenómeno migratorio, en el presente documento abonamos a la literatura al proponer un estudio a nivel agregado que nos permitirá evaluar qué tan cierto es que la inmigración ilegal afecta negativamente al país, estados o industrias estadounidenses. Esto es, los estudios que analizan el efecto de los flujos migratorios sobre las condiciones socioeconómicas del país receptor típicamente consideran datos desagregados. Dentro de ellos podemos mencionar los que son referencia en la literatura, incluyendo Borjas $(1987 ; 2006)$, Borjas et al. (1992) y Ottaviano y Peri (2012).

En contraste, aquí realizamos un estudio que explícitamente atiende las afirmaciones que alegan que la inmigración ilegal afecta las condi- 
ciones económicas en Estados Unidos a nivel agregado. Para ello, consideramos datos de aprehensiones en la frontera entre México y Estados Unidos como medida de inmigración ilegal, y el Producto Interno Bruto (PIB) agregado y desagregado por estados, así como datos de empleo en el ámbito industrial. Los estados e industrias que incluimos son aquellas que albergan al número más grande de inmigrantes sin documentos migratorios. El análisis de la inmigración con el PIB agregado tiene como objetivo identificar la relación temporal entre la actividad económica nacional y los flujos migratorios. Razonablemente, podríamos esperar que la inmigración siguiera la dinámica económica agregada, esto es, que el PIB funcione como un factor de atracción y preceda a los flujos migratorios; algo similar se podría anticipar con respecto al PIB estatal. Para el caso de las industrias, la relación que el empleo guarda con la inmigración no es tan directamente predecible. En particular, se podría esperar que, igualmente, las variaciones en el mercado laboral precedieran a la inmigración: cuando el empleo es robusto, los flujos migratorios aumentan, mientras que lo contrario es cierto cuando el empleo se debilita. Sin embargo, pudiera ser el caso que la inmigración ilegal de cierta forma influye sobre el mercado laboral de las industrias. Podría ser que la llegada de trabajadores "ilegales" produjera un efecto sobre el nivel de empleo. Si el argumento que formulan los medios conservadores estadounidenses sobre cómo los trabajadores "ilegales" desplazan a trabajadores nativos tiene sustento, tendría que ser el caso que la inmigración ilegal precede al empleo y se relaciona negativamente con él. En otras palabras, la inmigración ilegal daña las condiciones laborales de los trabajadores estadounidenses.

En este sentido, el presente análisis es cercano en espíritu a documentos que evalúan el efecto de la inmigración ilegal sobre variables económicas a nivel agregado. Sobre el tema, Liu (2010) estudia el efecto de los flujos de inmigración ilegal sobre el consumo; encuentra que en el largo plazo un incremento en el número de trabajadores sin documentos aumenta el consumo per cápita de los trabajadores nativos. Por su parte, Kiguhi y Mountford (2019) estiman el impacto de un aumento inesperado en inmigración sobre variables macroeconómicas que incluyen el PIB per cápita, consumo privado y desempleo. El efecto de la inmigración sobre estas variables es temporal y eventualmente regresa a su estado estacionario. 
Por consiguiente, notamos que el análisis y resultados obtenidos se entienden a nivel nacional, estatal y de industria, y no en un ámbito desagregado, por lo que nos será posible determinar la relación estocástica que la serie de inmigración guarda con variables agregadas; no obstante, determinar la influencia de la inmigración ilegal en sectores específicos de la sociedad o economía escapa del alcance de este ejercicio. Particularmente, supongamos que se determina que no existe una relación significativa entre la inmigración ilegal y la actividad económica en Arizona; esto no quiere decir que no exista afectación (o beneficio) de la inmigración sobre un sector económico específico en ese estado. Bien puede ser el caso que la inmigración conlleve un incremento en el consumo de artículos nostalgia, por ejemplo, en la ciudad de Yuma, y tenga un impacto positivo sobre los establecimientos comerciales en esa ciudad. Igualmente, es posible que encontremos que los flujos de inmigración ilegal no afectan el nivel de empleo en la industria de la construcción. Esto no implica que no existan empresas individuales en las que la llegada de trabajadores "ilegales" resulte en una disminución en el empleo de trabajadores nativos. Lo que estaríamos concluyendo es que, en el área industrial, el efecto de los flujos de inmigración ilegal no es estadísticamente significativo. Concedido el alcance de la presente investigación, creemos que resulta interesante verificar si la postura que señala a la inmigración ilegal como un mal en el ámbito económico agregado de Estados Unidos se valida en un ejercicio econométrico formal.

La estructura del documento es la siguiente: en la sección 1 se presentan los datos y se realiza una evaluación gráfica de los mismos. En la sección 2 se lleva a cabo el ejercicio econométrico y se discuten los resultados. La sección 3 concluye el documento.

\section{LOS DATOS}

El flujo de inmigración ilegal se mide por medio de las aprehensiones en la frontera México-Estados Unidos; esta medida se ha empleado en diversos documentos, incluyendo Hanson y Spilinbergo (1999) y Cornwell (2009). La información se obtuvo de las bases de datos del U.S. Department of Homeland Security. El PIB agregado y estatal se obtuvieron del Bureau of Economic Analysis. Los estados que se consideran en el estudio son California, Arizona, Nuevo México, Texas, Florida, Caro- 
lina del Norte, Illinois, Nueva York y Georgia, que, de acuerdo con el Migration Policy Institute, representan los de mayor población indocumentada. La actividad industrial se mide por medio del empleo en algunas de las industrias en las que los inmigrantes representan una proporción significativa de acuerdo al Pew Research Center: agricultura, ranchería, construcción, manufactura y ventas; los datos se obtuvieron del Bureau of Labor Statistics. Para todas las variables el periodo abarca de 1947 a 2015, excepto para el PIB estatal, que se encuentra disponible desde 1963. La periodicidad es anual y las cifras de PIB estatal se convirtieron en cifras reales y deflactaron el PIB nominal por el índice de precios al consumidor base 1982-1984.

En la Gráfica 1 se ilustra la serie de inmigración. El primer incremento significativo se observa entre 1950 y 1955 , que corresponde al periodo durante el cual el programa Bracero fue implementado. Después de una caída sustancial en la década de 1960, las aprehensiones repuntan para finales de 1970, y alcanzan su máximo alrededor de 1986, año en el que fue aprobada la reforma migratoria firmada por el ex presidente estadounidense Ronald Reagan. A partir del año 2000 se aprecia una caída sostenida, que se acentúa entre 2008 y 2010, muy probablemente debido a la crisis financiera que se experimentó en Estados Unidos y la recesión que le acompañó.

En la Gráfica 2 se presenta la tasa de crecimiento anual del PIB en Estados Unidos, conjuntamente con la tasa de crecimiento de la serie de inmigración. A primera vista no es posible identificar una relación clara entre las series, aunque se podrían mencionar algunos episodios interesantes. Por ejemplo, la caída sustancial de la inmigración alrededor de 1955 coincide con un incremento en el PIB; algo similar sucede entre 1960 y 1965. Durante la recesión de principios de 1980 se observa un estancamiento en la inmigración. Quizá lo que más llama la atención es que durante la recuperación económica en Estados Unidos, después de la Gran Recesión de 2009, la tasa de aprehensiones no aumenta, sino que disminuye para 2014 y 2015.

A partir de las observaciones de esta gráfica podríamos sospechar que el desempeño económico de Estados Unidos influye sobre los flujos de migración ilegal. Esta posibilidad será probada en el ejercicio econométrico que realizamos a continuación. 


\section{GRÁFICA 1}

NÚMERO DE APREHENSIONES EN LA FRONTERA ESTADOS UNIDOS-MÉXICO (1947-2015)

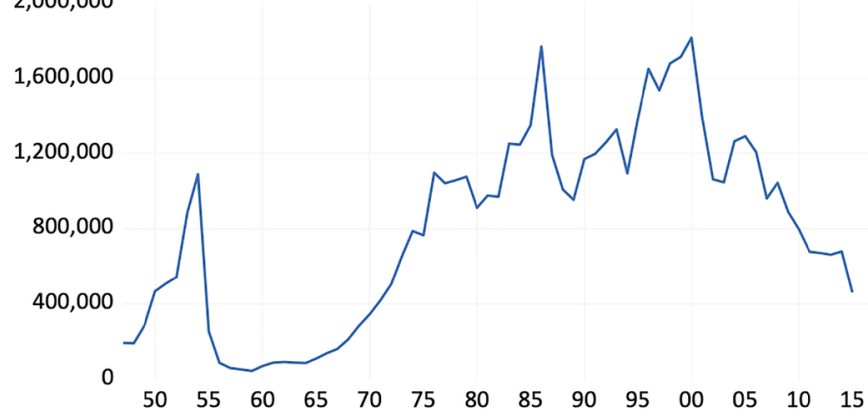

Fuente: elaborada con datos del U.S. Department of Homeland Security.

GRÁFICA 2

TASAS DE CRECIMIENTO DEL PIB DE ESTADOS UNIDOS Y LAS APREHENSIONES 1947-2015

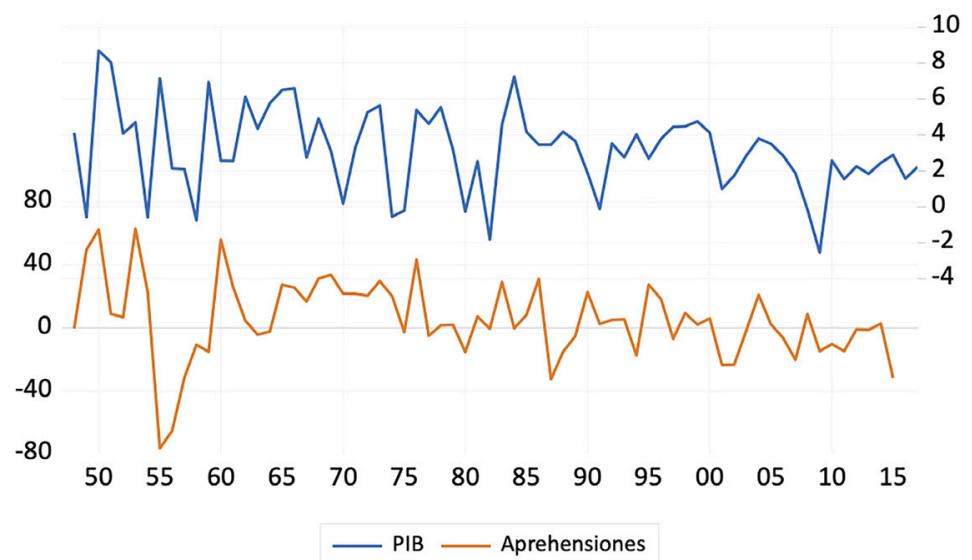

Fuente: elaborada con datos del U.S. Department of Homeland Security y el Bureau of Economic Analysis. 


\section{EJERCICIO ECONOMÉTRICO}

\subsection{Metodología}

La estrategia metodológica consiste, primero, en determinar el orden de integración de las variables de estudio y continúa con estimaciones de cointegración y ciclo común. Las pruebas de raíz unitaria que implementamos son estándar en los ejercicios de series de tiempo. Una de ellas se refiere a la propuesta de Kwiatkowski et al. (1992) (KPSS). La segunda es una prueba que considera cortes estructurales bajo el espíritu de Zivot y Andrews (1992), que permite generar estadísticos de prueba Dickey-Fuller modificados. Para el ejercicio de cointegración consideramos la metodología propuesta en Johansen (1991). La prueba de ciclo común sigue el procedimiento indicado en Vahid y Engle (1993).

Destacamos que, mientras existe una extensa gama de metodologías para llevar a cabo pruebas de cointegración y ciclo común, la que aquí implementamos presenta ventajas sobre alternativas como vectores de corrección de error o la aplicación de filtros, como se indica en Issler y Vahid (2001). Específicamente, la metodología de Vahid y Engle produce ganancias de eficiencia al imponer restricciones de corto y largo plazo en el sistema. Brevemente, una vez identificadas las series como integradas de orden 1, la secuencia inicia con la determinación de la existencia de cointegración. En caso de que las series exhiban una tendencia común, se procede a estimar las correlaciones canónicas entre ellas y realizar una prueba de hipótesis en la que se plantea la existencia de ciclo común. La prueba permite determinar la existencia de ciclo e identificar la relación de corto plazo que guardan las series. Formalmente, se considera un sistema de series de tiempo que se caracteriza de acuerdo con sus componentes de tendencia y ciclo, según Beveridge y Nelson (1981).

$$
y_{t}=C(1) \sum_{i=0}^{\infty} e_{t-i}+C^{*}(L) e_{t}
$$

El primer término del lado izquierdo de la expresión (1) corresponde al componente de tendencia y el segundo, al componente cíclico. La existencia de cointegración se sostendría si $\alpha^{\prime} C(1)=0$ donde la matriz $a$ contiene los $r$ vectores de cointegración. En nuestro ejercicio, dado que 
los sistemas que consideramos consisten de dos variables, $r$ puede ser a lo más igual a 1. Por otro lado, si fuera el caso que $\check{\alpha}^{\prime} C^{*}(L)=0$, entonces la matriz $\widetilde{\alpha}$ contendría los $s$ ciclos comunes. Una vez más, en nuestro caso el máximo de ciclos que podríamos encontrar es 1 . El vector de cointegración lo estimamos mediante la metodología de Johansen, mientras que para estimar el ciclo común primero se calculan las correlaciones canónicas al cuadrado $\left(\lambda_{j}^{2}\right)$ y enseguida se prueba la hipótesis $\lambda_{j}^{2}=0, \forall_{j}=$ $=1,2, \ldots, s$. El estadístico de prueba es

$$
C(p, s)=-(T-p-1) \sum_{i=1}^{s} \log \left(1-\lambda_{i}^{2}\right)
$$

donde $p$ es el número de rezagos óptimos obtenido en el ejercicio de cointegración. El estadístico se distribuye $\chi^{2}$ con grados de libertad igual a $s^{2}+s n p+s r-s n$, donde $s$ es el número de ciclos comunes en el sistema, $n$ es el número de variables, $r$ el número de vectores de cointegración y $p$ el número de rezagos óptimos. Finalmente, para determinar el efecto que la variable explicativa presenta sobre la variable de estudio, se estima la siguiente ecuación por medio de variables instrumentales generalizadas (GIV, por sus siglas en inglés):

$$
\Delta y_{t}=\beta \Delta x_{t}+\varepsilon_{t}
$$

donde el conjunto de instrumentos es el rezago de las variables contenidas en el sistema. En la siguiente sección seguimos esta secuencia para identificar la relación que guardan las series de actividad económica con la migración ilegal a Estados Unidos.

\subsection{Estimaciones}

El análisis empírico inicia con una evaluación de las propiedades de estacionariedad de las series. Los resultados de las pruebas KPSS y Dickey-Fuller modificada se presentan en el Cuadro 1. Para la totalidad de las series se encuentra un orden de integración de 1. Con base en la Gráfica 1, el nivel de la serie de aprehensiones parece exhibir una tendencia positiva de 1955 a 2000, mientras que el ciclo de la misma es evidentemente estacionario a juzgar por la ilustración de la Gráfica 2. Así, resulta razonable encontrar que la serie sea I(1). Para el caso del 
PIB de Estados Unidos, la literatura relevante sugiere que la serie es no estacionaria en niveles, pero estacionaria en primera diferencia. A pesar de que a la fecha continúa el debate sobre la naturaleza estocástica del PIB estadounidense, podemos afirmar que existe suficiente evidencia para afirmar en nuestro análisis que las series se encuentran integradas de orden 1 (véase, por ejemplo, Cushman 2016). Los PIB de los estados incluidos en el análisis se comportan de manera similar al PIB agregado, por lo que el resultado aquí obtenido es razonable. Respecto a las variables de empleo, las gráficas 3 y 4 muestran claramente que el nivel de las series de construcción, manufactura y ventas presentan una tendencia y que su ciclo es estacionario. Para las series de agricultura y ranchería, la evidencia de la Gráfica 5 no es tan contundente, mientras que desde el inicio de la muestra al año 2005 se aprecia una tendencia negativa; a partir de entonces parece que el empleo experimenta una expansión. Las primeras diferencias son visiblemente estacionarias (Gráfica 6). A pesar de la falta de claridad para estas series, tomamos los resultados de las pruebas estadísticas y concluimos que se encuentran integradas de orden 1.

CUADRO 1

PRUEBas de Raíz UNITARIA

\begin{tabular}{|c|c|c|c|c|c|}
\hline \multirow[t]{2}{*}{ Valores críticos } & \multicolumn{2}{|c|}{$\begin{array}{c}\text { Prueba KPSS } \\
5 \%: 0.146\end{array}$} & \multicolumn{3}{|c|}{$\begin{array}{l}\text { Prueba con corte estructural } \\
10 \%, 5 \% \text { y } 1 \%:-4.894,-5.176,-5.719\end{array}$} \\
\hline & Nivel & 1ra Diferencia & Nivel & 1ra Diferencia & Orden de Integración \\
\hline Aprehensiones & 0.129 & 0.078 & -2.638 & -7.639 & $\mathrm{I}(1)$ \\
\hline PIB USA & 0.238 & 0.055 & -3.057 & -7.753 & $\mathrm{I}(1)$ \\
\hline PIB California & 0.244 & 0.090 & -3.464 & -4.736 & $\mathrm{I}(1)$ \\
\hline PIB Arizona & 0.246 & 0.162 & -2.505 & -5.191 & I(1) \\
\hline PIB Nuevo México & 0.231 & 0.087 & -3.076 & -6.708 & $\mathrm{I}(1)$ \\
\hline PIB Texas & 0.228 & 0.072 & -3.739 & -7.125 & $\mathrm{I}(1)$ \\
\hline PIB Florida & 0.253 & 0.076 & -2.770 & -6.053 & $\mathrm{I}(1)$ \\
\hline PIB Georgia & 0.249 & 0.122 & -2.770 & -5.125 & $\mathrm{I}(1)$ \\
\hline PIB Carolina del Norte & 0.253 & 0.137 & -2.307 & -7.576 & I(1) \\
\hline PIB Nueva York & 0.243 & 0.084 & -2.983 & -6.686 & I(1) \\
\hline PIB Illinois & 0.254 & 0.097 & -1.901 & -7.204 & $\mathrm{I}(1)$ \\
\hline Agricultura & 0.082 & 0.048 & -4.119 & -11.199 & $\mathrm{I}(1)$ \\
\hline Ranchería & 0.088 & 0.049 & -4.407 & -11.446 & $\mathrm{I}(1)$ \\
\hline Construcción & 0.172 & 0.082 & -4.426 & -6.000 & $\mathrm{I}(1)$ \\
\hline Manufactura & 0.255 & 0.127 & -4.624 & -8.613 & $\mathrm{I}(1)$ \\
\hline Ventas & 0.243 & 0.085 & -5.212 & -7.746 & $\mathrm{I}(1)$ \\
\hline
\end{tabular}

Fuente: elaboración propia. 


\section{GRÁFICA 3}

EMPLEO EN CONSTRUCCIÓN, MANUFACTURA Y VENTAS.

SERIES EN NIVELES, 1947-2015

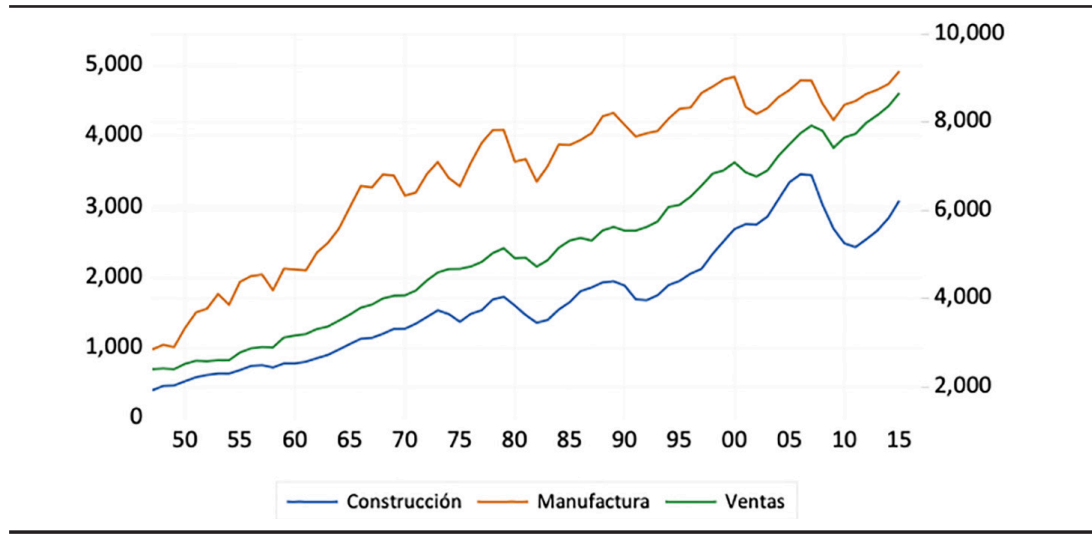

Fuente: elaborada con datos del Bureau of Labor Statistics.

\section{GRÁFICA 4}

EMPLEO EN CONSTRUCCIÓN, MANUFACTURA Y VENTAS. SERIES EN PRIMERAS DIFERENCIAS, 1947-2015

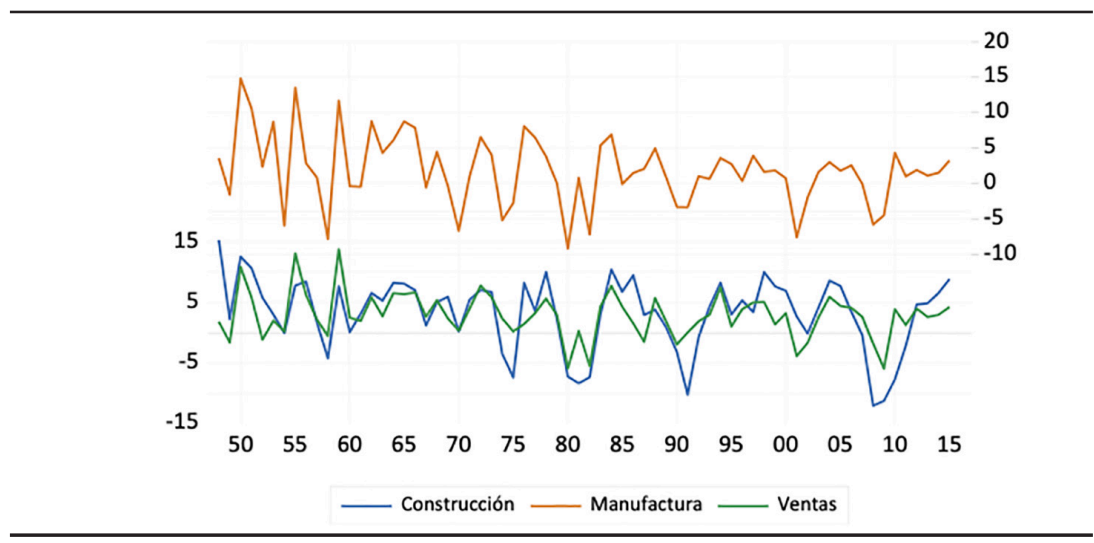

Fuente: elaborada con datos del Bureau of Labor Statistics. 


\section{GRÁFICA 5}

EMPLEO EN AgRICULtURA y RANCHERÍA. SeRIES EN NIVELES 1947-2015

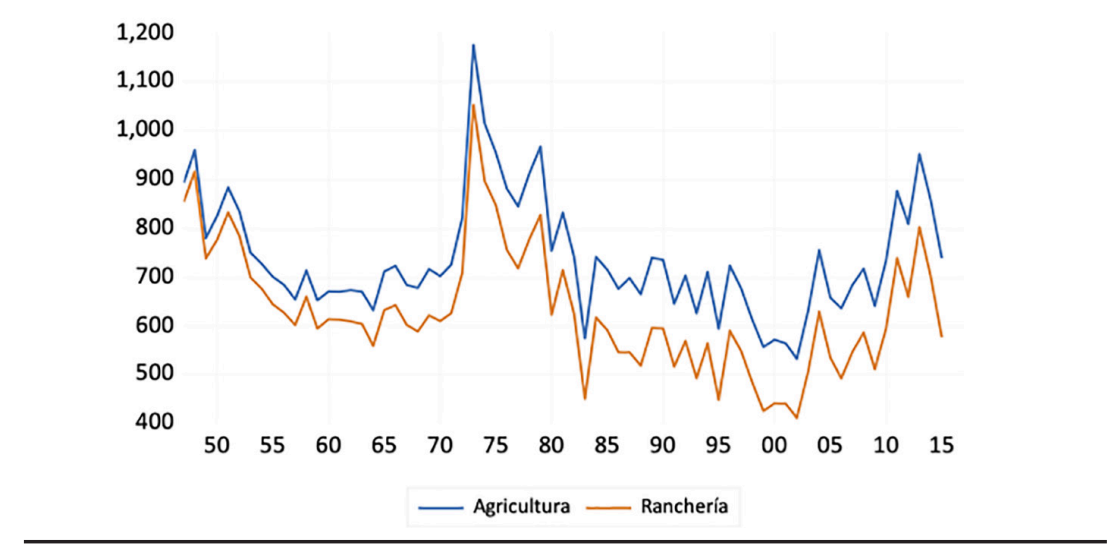

Fuente: elaborada con datos del Bureau of Labor Statistics.

\section{GRÁFICA 6}

EMPLEO EN AGRICULTURA Y RANCHERÍA. SERIES EN PRIMERAS DIFERENCIAS 1947-2015

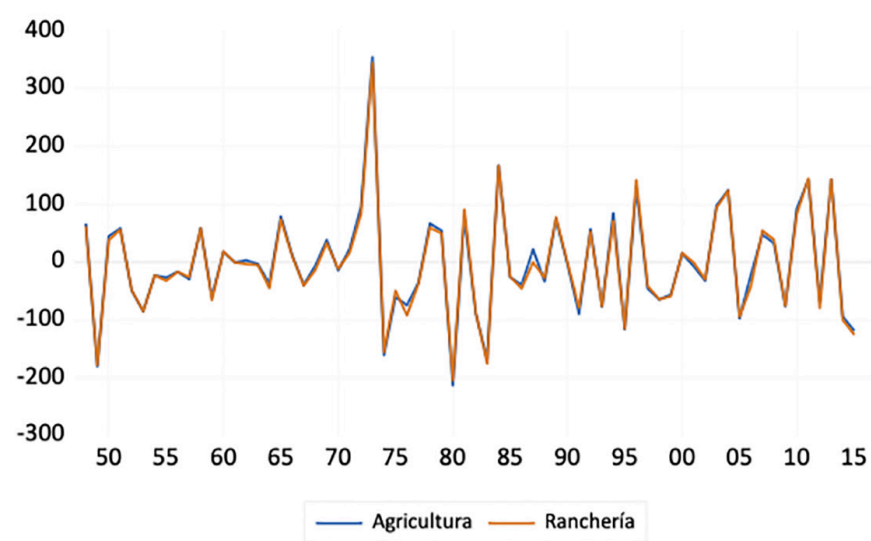

Fuente: elaborada con datos del Bureau of Labor Statistics. 
Los resultados de las pruebas de cointegración se presentan en el Cuadro 2. La serie de aprehensiones comparte una tendencia común con cada una de las series de producción, tanto a nivel agregado como estatal. Debido a que normalizamos la serie de aprehensiones, podríamos interpretar los resultados como evidencia de que la actividad económica en Estados Unidos atrae la migración en el largo plazo: mejoras en el desempeño económico se relacionan con incrementos en la inmigración, mientras que lo opuesto es cierto cuando la actividad económica se debilita. En cuanto al empleo en el ámbito industrial, se encuentra igualmente una relación positiva y significativa de largo plazo. Destaca que los coeficientes más elevados corresponden a la construcción y manufactura. Este resultado es consistente con los datos del Pew Research Center, que señalan que el porcentaje de trabajadores indocumentados empleados en estas industrias, respecto al número total de trabajadores indocumentados, es mayor que el mismo en cuanto a la población de trabajadores nacidos en Estados Unidos. En general, encontramos que estos resultados son consistentes con la teoría de atracción, que sugiere que la actividad económica en el país receptor funciona como un factor que influye sobre la decisión de migrar, siendo esta relación positiva.

CUADRO 2

PRUEBAS DE COINTEGRACIÓN

\begin{tabular}{|ccc|}
\hline Sistemas & Probabilidad & $\begin{array}{c}\text { Vector de } \\
\text { cointegración }\end{array}$ \\
\hline Aprehensiones con & & $1,0.80$ \\
\hline PIB USA & 0.000 & $1,0.64$ \\
\hline PIB California & 0.002 & $1,0.56$ \\
\hline PIB Arizona & 0.004 & $1,0.28$ \\
\hline PIB Nuevo México & 0.000 & $1,0.54$ \\
\hline PIB Texas & 0.002 & $1,0.56$ \\
\hline PIB Florida & 0.003 & $1,0.24$ \\
\hline PIB Georgia & 0.000 & $1,0.28$ \\
\hline PIB Carolina del Norte & 0.000 & $1,0.44$ \\
\hline PIB Nueva York & 0.000 & $1,0.41$ \\
\hline PIB Illinois & 0.000 & $1,0.33$ \\
\hline Agricultura & & $1,0.53$ \\
\hline Ranchería & 0.078 & $1,1.87$ \\
\hline Construcción & 0.071 & $1,1.97$ \\
\hline Manufactura & 0.044 & $1,1.25$ \\
\hline Ventas & 0.047 & \\
\hline
\end{tabular}

Fuente: elaboración propia. 
Para la evaluación de la relación cíclica entre las variables se realizan dos ejercicios. El primero consiste en determinar la existencia de ciclo común entre las series siguiendo la metodología descrita anteriormente. El propósito de este ejercicio es precisar si las fluctuaciones de las variables económicas coinciden con las de la inmigración ilegal. De encontrarse un ciclo común, sabríamos que ambas series se "mueven juntas" en el corto plazo. En el segundo ejercicio procedemos a estimar la ecuación 3, que aparece en el apartado metodológico, con el fin de identificar el efecto que la inmigración ilegal presenta sobre la actividad económica. Para cumplir con este objetivo, estimamos dos especificaciones: una considera la serie de aprehensiones como variable dependiente y cada una de las series de actividad económica como explicativa. La otra invierte el orden de las variables, de tal forma que la variable de aprehensiones explica a la actividad económica. ¿Qué resultados esperamos encontrar? Razonablemente, siguiendo las teorías de migración establecidas, las variables económicas tendrían que influir sobre la inmigración ilegal de manera positiva, siendo esto evidencia del efecto de atracción que la economía de las comunidades receptoras ejerce sobre la migración. Pero, por otro lado, si los argumentos de los medios conservadores sobre el daño que la inmigración ilegal produce en la economía de Estados Unidos tienen sustento, encontraríamos que las aprehensiones afectan a las condiciones económicas de manera negativa y significativa.

Los resultados de las estimaciones se presentan en el Cuadro 3. La columna "Probabilidad" reporta la posibilidad de que exista un ciclo común entre las series. Se aprecia que el ciclo de las aprehensiones coincide con el de los PIB de California, Arizona, Florida, Georgia y Carolina del Norte a 5 por ciento. El hallazgo tiene sentido ya que estos estados reciben cantidades significativas de inmigrantes, que muy probablemente ponen atención a las condiciones económicas de estas entidades cuando toman la decisión de migrar. Interesantemente, no se encuentra un ciclo común entre las aprehensiones y el PIB de Estados Unidos. En principio, hubiéramos esperado que las fluctuaciones cíclicas de la economía norteamericana se relacionaran con las fluctuaciones migratorias. Después de todo, la evidencia anecdótica sugiere que los migrantes siguen de cerca el desempeño económico del país cuando determinan el momento de migrar. Durante la Gran Recesión de 2009, por ejemplo, un número significativo de migrantes regresó 
a sus comunidades de origen, y el número de aquellos que deseaban aventurarse a la frontera disminuyó. A pesar de que nuestro hallazgo es inesperado, podríamos explicarlo si revisamos los antecedentes sobre el fenómeno de la inmigración ilegal en la literatura existente. Notamos que Hanson y Spilbergo (1999) identifican la diferencia salarial entre Estados Unidos y México como el factor que mayormente influye sobre la decisión de migrar. Más adelante revisaremos este resultado puntualmente.

Respecto a las series de empleo, no encontramos evidencia de la existencia de un ciclo común con la serie de inmigración, lo cual sugiere que los movimientos cíclicos de los flujos migratorios no coinciden con la dinámica del mercado laboral en las industrias que aquí analizamos.

Para la estimación GIV se consideran dos sistemas con el propósito de identificar cuál serie presenta un efecto sobre la otra: uno en el que la variable dependiente es la serie de aprehensiones y otro en el que la variable dependiente es la serie económica. Así, en el cuadro se reportan dos filas para cada serie de activad económica, una que se etiqueta "Explicativa" (la variable económica es la variable independiente) y otra "Dependiente" (la variable económica es la variable dependiente). La única excepción es el PIB agregado, ya que razonablemente no se esperaría que los flujos de inmigración ilegal influyan sobre el PIB de Estados Unidos significativamente. Para esta variable únicamente se considera al PIB como variable explicativa de las aprehensiones.

Lo primero que destacamos en el Cuadro 3 es que la probabilidad de la existencia de un ciclo común permanece constante, independientemente de cuál de las series se postula como dependiente. Esto es intuitivo, pues la dinámica cíclica de las series no depende de cuál de las series se modele como dependiente. En cuanto a los coeficientes, primero nos enfocamos en los resultados de las filas en las que aparece la palabra "Explicativa", que son los sistemas en los que la variable dependiente es la serie de inmigración. Notamos que en todos los casos para los que se encuentra la existencia de un ciclo común (PIB de California, Arizona, Florida, Georgia, y Carolina del Norte), el coeficiente de la regresión es significativo, con excepción del PIB de Carolina del Norte. Los PIB de California y Arizona reportan los coeficientes de mayor magnitud. Este resultado sugiere que, cuando la actividad económica en California, Arizona, Florida y Georgia se expande (contrae), los flujos de inmigración ilegal aumentan (disminuyen), lo cual confirma 
que el desempeño de la economía funciona como un factor de atracción de migración. Este argumento no es posible formularlo respecto a las series de empleo, pues en ningún caso el coeficiente resultó significativo. Quizá podríamos entender este resultado si reconocemos que la información sobre el nivel de empleo industrial no es ampliamente conocida por quienes pretenden emigrar, al menos no al grado de la información sobre las condiciones económicas de los estados. Específicamente, es probable que los migrantes se enteren de cómo se encuentra la economía estatal, pero no de las fluctuaciones en el nivel de empleo en industrias específicas. Ésta, por supuesto, es sólo una sugerencia que pudiera explicar el resultado.

En cuanto a las filas donde aparece la palabra "Dependiente", que se refieren a las estimaciones en las que la variable dependiente es la serie económica, encontramos que en ningún caso el coeficiente es significativo. Así, es claro que la inmigración ilegal, medida por las aprehensiones en la frontera, no influye significativamente sobre las fluctuaciones económicas de los estados o la dinámica laboral de las industrias aquí examinadas. Queda establecido entonces que las afirmaciones y reclamos del gobierno federal y medios conservadores en Estados Unidos sobre el daño económico que causan los inmigrantes "ilegales" no tienen sustento. No es posible afirmar que la inmigración ilegal afecta significativa y negativamente a los estados o industrias donde se ubica el mayor número de trabajadores sin documentación migratoria adecuada. Mientras que puede ser el caso que algún trabajador sea desplazado por un inmigrante en alguna de las industrias que analizamos, no se puede generalizar y afirmar que los inmigrantes "le quitan el trabajo" a los estadounidenses. Estadísticamente no existe evidencia de que el efecto sea significativo.

Más allá del análisis formal, sostenemos que los resultados que aquí presentamos son razonables. Si consideramos el tamaño de las economías e industrias analizadas, resulta poco probable que la llegada de trabajadores "ilegales" afecte su desempeño significativamente. El efecto marginal de que un trabajador sin documentos migratorios adecuados se sume a las actividades productivas de un estado o industria es nulo. Sospechamos que la mayoría de personas de ciencia coincidirían con nuestra apreciación. Nos gustaría creer que la mayoría de personas que habitan en Estados Unidos igualmente aceptarían este argumento, puesto que tiene sentido. Desafortunadamente, existe un 
número importante de individuos en ese país que toman los señalamientos del gobierno federal y de comentaristas conservadores como verdad absoluta, e insisten en condenar a los inmigrantes como personas que lastiman las condiciones económicas de las comunidades que los reciben.

CUADRO 3

PRUEBAS DE CICLO COMÚN: SERIES CONTEMPORÁNEAS

\begin{tabular}{|c|c|c|c|}
\hline Sistemas & Probabilidad & Estadístico $\mathrm{t}$ & Coeficiente \\
\hline $\begin{array}{c}\text { Aprehensiones con } \\
\text { PIB USA }\end{array}$ & 0.016 & 0.596 & 0.009 \\
\hline $\begin{array}{c}\text { PIB California Explicativa } \\
\text { Dependiente }\end{array}$ & 0410 & $\begin{array}{l}2.413 \\
1.178\end{array}$ & $\begin{array}{l}0.340 \\
1.705\end{array}$ \\
\hline $\begin{array}{l}\text { PIB Arizona Explicativa } \\
\text { Dependiente }\end{array}$ & 0.228 & $\begin{array}{l}2.087 \\
1.404\end{array}$ & $\begin{array}{l}0.349 \\
1.125\end{array}$ \\
\hline $\begin{array}{l}\text { PIB N. México Explicativa } \\
\text { Dependiente }\end{array}$ & 0.038 & $\begin{array}{l}1.318 \\
1.366\end{array}$ & $\begin{array}{l}0.092 \\
2.104\end{array}$ \\
\hline $\begin{array}{l}\text { PIB Texas Explicativa } \\
\text { Dependiente }\end{array}$ & 0.040 & $\begin{array}{l}2.060 \\
1.594\end{array}$ & $\begin{array}{l}0.140 \\
2.015\end{array}$ \\
\hline $\begin{array}{l}\text { PIB Florida Explicativa } \\
\text { Dependiente }\end{array}$ & 0.110 & $\begin{array}{l}2.270 \\
1.594\end{array}$ & $\begin{array}{l}0.237 \\
1.523\end{array}$ \\
\hline $\begin{array}{l}\text { PIB Georgia Explicativa } \\
\text { Dependiente }\end{array}$ & 0.128 & $\begin{array}{l}1.851 \\
1.158\end{array}$ & $\begin{array}{l}0.187 \\
1.295\end{array}$ \\
\hline $\begin{array}{c}\text { PIB Carolina del Norte } \\
\text { Explicativa } \\
\text { Dependiente }\end{array}$ & 0.051 & $\begin{array}{l}1.379 \\
1.055\end{array}$ & $\begin{array}{l}0.103 \\
1.515\end{array}$ \\
\hline $\begin{array}{l}\text { PIB Nueva York Explicativa } \\
\text { Dependiente }\end{array}$ & 0.029 & $\begin{array}{l}-0.749 \\
-0.660\end{array}$ & $\begin{array}{l}-0.047 \\
-1.193\end{array}$ \\
\hline $\begin{array}{l}\text { PIB Illinois Explicativa } \\
\text { Dependiente }\end{array}$ & 0.009 & $\begin{array}{l}0.380 \\
0.350\end{array}$ & $\begin{array}{l}0.019 \\
0.687\end{array}$ \\
\hline $\begin{array}{l}\text { Agricultura Explicativa } \\
\text { Dependiente }\end{array}$ & 0.024 & $\begin{array}{l}-0.132 \\
-0.195\end{array}$ & $\begin{array}{l}-0.012 \\
-0.185\end{array}$ \\
\hline $\begin{array}{l}\text { Ranchería Explicativa } \\
\text { Dependiente }\end{array}$ & 0.011 & $\begin{array}{l}-0.298 \\
-0.394\end{array}$ & $\begin{array}{l}-0.034 \\
-0.285\end{array}$ \\
\hline $\begin{array}{c}\text { Construcción Explicativa } \\
\text { Dependiente }\end{array}$ & 0.000 & $\begin{array}{l}0.229 \\
0.189\end{array}$ & $\begin{array}{l}0.010 \\
0.196\end{array}$ \\
\hline $\begin{array}{c}\text { Manufactura Explicativa } \\
\text { Dependiente }\end{array}$ & 0.004 & $\begin{array}{l}0.182 \\
0.250\end{array}$ & $\begin{array}{l}0.006 \\
0.499\end{array}$ \\
\hline $\begin{array}{l}\text { Ventas Explicativa } \\
\text { Dependiente }\end{array}$ & 0.003 & $\begin{array}{l}0.028 \\
0.033\end{array}$ & $\begin{array}{l}0.001 \\
0.082\end{array}$ \\
\hline
\end{tabular}

Fuente: elaboración propia.

Finalmente, quisimos reevaluar la relación de la producción con la inmigración ilegal, pues el resultado de la no existencia de un ciclo común entre el PIB agregado y la serie de inmigración nos llamó la 
atención. Razonablemente, podríamos haber esperado que el ciclo de las aprehensiones siguiera al ciclo económico. La Gráfica 7 muestra las tasas de crecimiento de ambas series con la escala sobrepuesta para apreciar de manera más clara la relación cíclica entre ellas. Es evidente que los ciclos no coinciden; parece, sin embargo, que el ciclo del PIB precede al ciclo de las aprehensiones. Esto es claro entre 1955 y 1960, en 1975, a principios de la década de 1980 y durante las crisis de 2001 y 2009. Así, resulta pertinente postular que, quizá, si se rezaga el ciclo del PIB, se pudiera encontrar un ciclo común con el ciclo de las aprehensiones. En el Cuadro 4 aparecen los resultados de la prueba de ciclo común para la serie del PIB rezagada de 1 a 3 periodos. Nótese que a medida que aumentan los rezagos, la probabilidad de un ciclo común crece; para 3 rezagos ya se encuentra evidencia de un ciclo común a 9.5 por ciento. Esto quiere decir que la migración ilegal responde al ciclo económico de Estados Unidos con aproximadamente tres periodos de rezago. Específicamente, no es el caso que cuando las condiciones económicas de ese país mejoran (empeoran), instantáneamente aumenta (disminuye) el flujo migratorio, sino que demora. De acuerdo con los resultados, la migración responde al tercer periodo. Este hallazgo es intuitivo. Cuando las personas se enteran de que la economía de Estados Unidos ha entrado en un periodo de auge, por ejemplo, típicamente se preparan para realizar el viaje. Seguramente toma tiempo llevar a cabo los preparativos prudentes para estar en condición de aventurarse a la frontera.

En el Cuadro 4 también se reportan los resultados de la regresión que evalúa el efecto del PIB sobre las aprehensiones. Para todos los rezagos el coeficiente no es significativo. Una vez más, esto es consistente con el argumento de que existen factores, además del PIB, que influyen sobre la migración ilegal. Como se mencionó, el diferencial salarial puede ser uno de ellos, o simplemente las redes de familiares y conocidos con los que los migrantes cuentan en Estados Unidos. 


\section{GRÁFICA 7}

TASA DE CRECIMIENTO ANUAL DEL PIB Y LAS APREHENSIONES 1947-2015

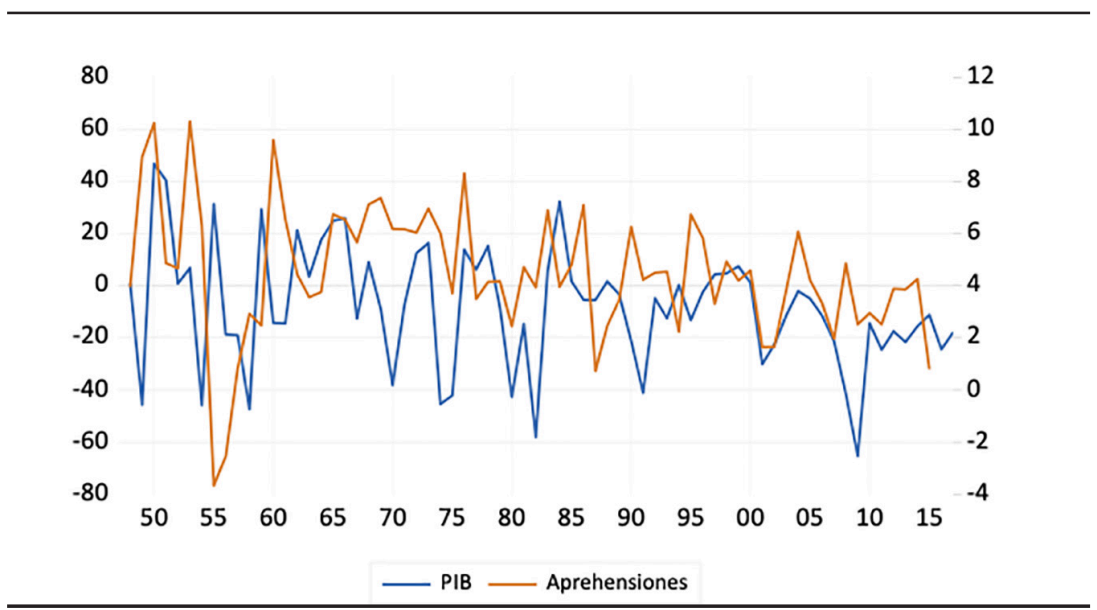

Fuente: elaborada con datos del Bureau of Labor Statistics.

CUADRO 4

PRUEBAS DE CICLO COMÚN: SERIES REZAGADAS

\begin{tabular}{cccc}
\hline Sistemas & Probabilidad & Estadístico t & Coeficiente \\
\hline Aprehensiones con & & & \\
\hline PIB de EUA & & & 0.016 \\
Rezagado 1 periodo & 0.025 & 1.030 & 0.021 \\
Rezagado 2 periodos & 0.038 & 1.299 & -0.010 \\
\hline Rezagado 3 periodos & 0.095 & -0.623 & \\
\hline
\end{tabular}

Fuente: elaboración propia.

\section{CONCLUSIONES}

El ambiente hostil que la comunidad inmigrante en Estados Unidos vive actualmente es resultado, en gran medida, de la actitud que la administración federal -concluída en 2020- tomó hacia ella. Desde el inicio de su candidatura, y hasta la fecha, el ex presidente estadounidense caracterizó a los inmigrantes, particularmente aquellos de origen mexicano, como personas indeseables. Comentaristas conservadores se sumaron a los ataques y cubrieron constantemente notas en las que mostraban a los inmigrantes como vándalos, que cometen crímenes, abusan del sistema de apoyo social y no abonan positivamente a la comunidad. Un reclamo 
que frecuentemente se escucha en medios conservadores es que los inmigrantes afectan negativamente las condiciones económicas de las comunidades receptoras. Pero, ¿qué tan cierto es esto? ¿Existe evidencia de que la llegada de inmigrantes produzca un costo económico significativo a nivel agregado? El análisis que aquí presentamos ofrece una respuesta contundente: NO. No hay evidencia a nivel del PIB nacional, estatal y de empleo industrial de que la inmigración ilegal afecte su dinámica. Lo que sí confirmamos es que las condiciones económicas sirven como un factor de atracción para la migración, resultado que ya esperábamos, pues es ampliamente aceptado que el estado económico del lugar de destino influye sobre la decisión de migrar. Podemos entonces afirmar categóricamente que los ataques hacia la comunidad inmigrante en Estados Unidos carecen de sustento. Los comentaristas conservadores toman un caso particular y lo aplican a la generalidad de inmigrantes. Hacen referencia a un inmigrante "ilegal" que cometió un crimen y se refieren a todos los inmigrantes como criminales. Hacen referencia a una empresa donde un trabajador "ilegal" tomó el empleo de un trabajador estadounidense y reclaman que los "ilegales" les están quitando el empleo a los nativos. Desafortunadamente, la audiencia del ex presidente y de estos medios conservadores cree que lo que les están transmitiendo es cierto. Lo que aquí mostramos es que no lo es. A nivel agregado y estadísticamente hablando, la inmigración ilegal no afecta negativamente las condiciones económicas. Finalmente, destacamos que los resultados que aquí presentamos llaman a profundizar en el análisis. Podría ser interesante, por ejemplo, realizar un estudio similar a éste, pero considerar series más desagregadas, como producto a nivel de municipalidad o series de crimen o violencia a nivel de ciudad. Sería también prudente evaluar las industrias a nivel estatal, ya que la actividad agrícola, por ejemplo, no es homogénea a través de diferentes estados; la agricultura en California no es de igual importancia a la de Nueva York. Ciertamente estaremos atendiendo alguna de estas posibilidades en el futuro. 


\section{REFERENCIAS}

Altonji, Joseph y David Card (1991), "The Effects of Immigration on the Labor Market Outcomes of Less-Skilled Natives", en J.M. Abowd y R.B. Freeman (eds.), Immigration, Trade, and the Labor Market, The University of Chicago Press, Chicago.

Beveridge, Stephen y Charles Nelson (1981), “A New Approach to Decomposition of Economic Time Series into Permanent and Transitory Components with Particular Attention to the Measurement of the Business Cycle", Journal of Monetary Economics, 7 (2), pp. 151-174.

Borjas, George (1987), "Self-selection and the Earnings of Immigrants", American Economic Review, 77 (4), pp. 531-553.

Borjas, George (2003), "The Labor Demand Curve is Downward Sloping: Reexamining the Impact of Immigration on the Labor Market", Quarterly Journal of Economics, 118 (4), pp. 1335-1374.

Borjas, George (2006), "Native Internal Migration and the Labor Market Impact of Immigration", Journal of Human Resources, XLI (2), pp. 221-258.

Borjas, George (2011), "Poverty and Program Participation among Immigrant Children", The Future of Children, 21 (1), pp. 247-266.

Borjas, George, Stephen Bronas y Stephen Trejo (1992), "Self-selection and Internal Migration in the United States", Journal of Urban Economics, 32 (2), pp. 59-185.

Cornwell, Derekh (2009), "Exploratory Time Series Analysis of Apprehensions and Linewatch Hours on the Southwest Border", EUA, Office of Immigration Statistics, DIANE Publishing.

Cushman, David (2016), “A Unit Root in Postwar U.S. Real GDP Still Cannot Be Rejected, and Yes, It Matters", Econ Journal Watch, 13 (1), pp. 5-45.

Hanson, Gordon. H. y Antonio Spilimbergo (1999), "Illegal Immigration, Border Enforcement, and Relative Wages: Evidence from Apprehensions at the U.S.-Mexico Border", American Economic Review, 89 (5), pp. 1337-1357.

Issler, Joao V. y Farshid Vahid (2001), "Common Cycles and the Importance of Transitory Shocks to Macroeconomic Aggregates", Journal of Monetary Economics, 47 (3), pp. 449-475.

Johansen, Soren (1991), "Estimation and Hypothesis Testing of Cointegration Vectors in Gaussian Vector Autoregressive Models", Econometrica: Journal of the Econometric Society, 59 (6), pp. 1551-1580.

Kiguchi, Takehiro y Andrew Mountford (2019), "Immigration and Unemployment: A Macroeconomic Approach", Macroeconomic Dynamics, 23 (4), pp. 1313-1339.

Kwiatkowski, Denis, Peter Phillips, Peter Schmidt y Yongcheol Shin (1992), "Testing the Null Hypothesis of Stationarity against the Alternative of a Unit Root: How Sure are We that Economic Time Series have a Unit Root?", Journal of Econometrics, 54 (1-3), pp. 159-178. 
Liu, Xiangbo (2010), “On the Macroeconomic and Welfare Effects of Illegal Immigration", Journal of Economic Dynamics and Control, 34 (12), pp. 2547-2567.

Mandelman, Federico y Andrei Zlate (2012), "Immigration, Remittances, and Business Cycles”, Journal of Monetary Economics, 59 (2), pp. 196-213.

Ottaviano, Gianmarco y Giovanni Peri (2012), "Rethinking the Effect of Immigration on Wages", Journal of the European Economic Association, 10 (1), pp. 152-197.

Peri, Giovanni (2016), "Immigration, Productivity, and Labor Markets", Journal of Economic Perspectives, 30 (4), pp. 3-30.

Peri, Giovanni y Chad Sparber (2009), "Trade Specialization, Immigration, and Wages”, American Economic Journal: Applied Economics, 1 (3), pp. 135169.

Vahid, Farshid y Robert Engle (1993), "Common Trends and Common Cycles", Journal of Applied Econometrics, 8 (4), pp. 341-360.

Zivot, Eric y Donald Andrews (1992), "Further Evidence on the Great Crash, the Oil-Price Shock, and the Unit-Root Hypothesis", Journal of Business \& Economic Statistics, 10 (3), pp. 251-270. 